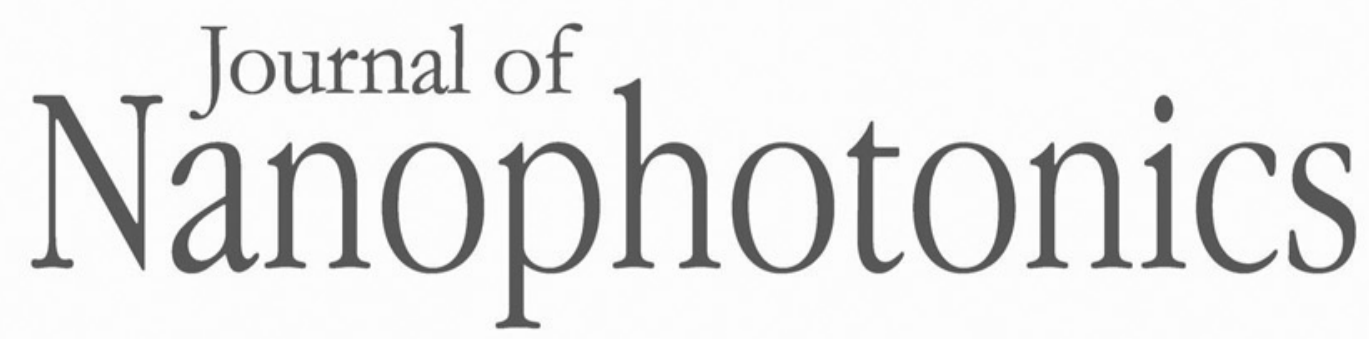

Nanophotonics.SPIEDigitalLibrary.org

\title{
Special Section Guest Editorial: Nanocarbon Photonics and Optoelectronics
}

Sergey Maksimenko

Yuri Svirko 


\title{
Special Section Guest Editorial: Nanocarbon Photonics and Optoelectronics
}

\author{
Sergey Maksimenko a and Yuri Svirko ${ }^{\mathrm{b}}$ \\ ${ }^{a}$ Belarusian State University, Institute for Nuclear Problems, Minsk, Belarus \\ ${ }^{b}$ University of Eastern Finland, Institute of Photonics, Joensuu, Finland
}

This special section of the Journal of Nanophotonics covers synthesis and properties of carbonbased nanostructures with reduced dimensionality with focus on materials for photonic and optoelectronic applications. All papers have been presented at the Fifth International Workshop on Nanocarbon Photonics and Optoelectronics (NPO2016), which was held in the Saimaa Holiday Club, Lappeenranta, Finland, 1-6 August 2016. The workshop was chaired by Professors Esko Kauppinen (Aalto University, Helsinki, Finland), Alexander Obraztsov (M.V. Lomonosov Moscow State University, Moscow, Russia) and Yuri Svirko (University of Eastern Finland, Joensuu, Finland). NPO2016 continues a series of meetings organized by the University of Eastern Finland (www.npo.fi). Since 2008, NPO workshops have brought together researchers from academy and industry to discuss fundamental and application properties of carbon-based and other low-dimensional materials.

NPO workshops offer a highly scientific and creative and friendly atmosphere as well as unique opportunities for communication for researchers working in diverse fields of lowdimensional materials. NPO2016 was composed of lectures and invited talks presented by leading scientists who provide comprehensive reviews of the state of the art, as well as oral and poster presentations of young researchers working in the rapidly developing field of photonics and optoelectronics of low-dimensional materials.

This special section covers synthesis and characterization of nanocarbon materials (A.B. Loginov and R.R. Ismagilov), nonlinear optical phenomena in nanoparticles (K.G. Mikheev et al., V.V. Vanyukov et al., and E. S. Babich), microwave and THz photonics and optoelectronics with carbon materials (P.P. Kuzhir et al., P. A. Kanygin et al.), and photovoltaics (A.S. Saushin et al., E. Karimi and S.M.B. Ghorashi).

In NPO2016, special attention was paid to magnetic properties of graphene-related materials and in particular to the research activity of the international team led by Dr. Tatiana Makarova, a globally recognized authority in the area. In the special section, these results are presented by papers by I. Zakharchuk et al. and A. Komlev et al. Unfortunately Dr. Makarova passed away on 7 September 2016, and NPO2016 was the last international conference in which she took part. All participants, and especially young researchers, will remember her great carriage in science, her determination, and her strong personality.

We hope that this special section will be of interest for the nanophotonics community in bringing new ideas and attracting more attention to the exciting area of nanocarbon photonics and optoelectronics.

(1) 2017 Society of Photo-Optical Instrumentation Engineers (SPIE) 